\title{
Signal-amplified Immunoassay Based on Biometallization of Palladium Nanoparticles and Nickel-Phosphorus Enhancement
}

\author{
Xinli Guo, Ping Zhang, Xinliang Liu, Na Zhang, Meihua Jiang, Qi Kang "'Dazhong Shen* \\ College of Chemistry, Chemical Engineering and Materials Science, Collaborative Innovation Center \\ of Functionalized Probes for Chemical Imaging in Universities of Shandong, Key Laboratory of \\ Molecular and Nano Probes, Ministry of Education, Shandong Provincial Key Laboratory of Clean \\ Production of Fine Chemicals, Shandong Normal University, Jinan 250014, P. R. China. \\ *E-mail:qikang@sdu.edu.cn; dzshen@sdnu.edu.cn
}

doi: $10.20964 / 2017.04 .36$

Received: 14 December 2016 / Accepted: 12 February 2017 / Published: 12 March 2017

\begin{abstract}
A sensitive electrochemical immunosensor was reported with signal amplification by biometallization of Ni-P. In this strategy, the primary antibody probes were immobilized on the aminated glass surface to bond the target antigen. Bounding with alkaline phosphatase (ALP) conjugated second antibody, the sandwich-type immunocomplex was formed. The signal tag of ALP in the immunocomplex can catalyze its substrate of $p$-aminophenyl phosphate to produce $\mathrm{p}$-aminophenol, which can reduce $\operatorname{Pd}(\mathrm{II})$ in solution to Pd nanocrystals onto the surface. The Pd nanocrystals were served as the catalyst for electroless deposition of Ni-P layer in a successive signal amplification stage. Dissolved by HNO3, the amount of Ni deposited was determination by adsorptive stripping voltammetry method to quantify the target antigen. With human IgG as the model antigen, the applicability of the method was exploited. By using a bismuth nanoparticles modified glassy carbon electrode for Ni determination, the effect of the concentrations of $p$-aminophenol and $\mathrm{Pd}(\mathrm{II})$, biometallization time and temperature on the electroless deposition Ni-P were investigated and optimized. The stripping peak current of Ni was proportional to the concentration of human $\mathrm{IgG}$ in a dynamic range of $0.1-100 \mathrm{pg} / \mathrm{mL}$ with a detection limit of $0.03 \mathrm{pg} / \mathrm{mL}$.
\end{abstract}

Keywords: Immunosensor; biometallization, Ni-P enhancement; stripping voltammetry

\section{$\underline{\text { FULL TEXT }}$}

(C) 2017 The Authors. Published by ESG (www.electrochemsci.org). This article is an open access article distributed under the terms and conditions of the Creative Commons Attribution license (http://creativecommons.org/licenses/by/4.0/). 